\title{
Optimization design of electric vehicle charging pile based on coil
}

\section{magnetic resonance coupling control}

\author{
Xu Jian-Rui ${ }^{\mathrm{a}}$, Lu Xing-Hua ${ }^{\mathrm{b},{ }^{*}}$, Ye Ming-Ming ${ }^{\mathrm{c}},{ }$ Xue Jlan-Xin ${ }^{\mathrm{d}}$ \\ Huali College Guangdong University of Technology, Guangzhou Zengcheng, 511325, P. R. China

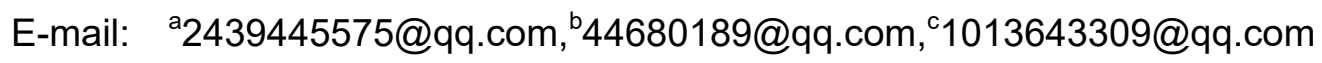 \\ , $13421024461 @ 163 . c o m$
}

\begin{abstract}
The charging pile is influenced by electromagnetic coupling interference factors of primary coil and secondary coil of the electric vehicle charging, resulting in excessive demand on the parking position, in order to solve this problem, a power transmission optimization control technique of electric car charging pile is proposed based on coil magnetic resonance coupling. The construction of electric vehicle coupling coil charging pile is taken with spiral planar coil, the half bridge LLC resonant inverter is designed as the charging inverter control unit, the resonant frequency, mutual inductance, resistance and other parameters are analyzed for charging system for electric energy transmission efficiency, and as a basis for parameter optimization design of charging pile coil series, coupled control coil magnetic resonance is taken near resonance, the electricity charging pile optimization design of transmission system is realized. The test results show that using the method of design of electric vehicle charging pile, it has high efficiency of power transmission, the stable output voltage is achieved in a large load range, output power has high gain.
\end{abstract}

Keywords: electric vehicles; charging pile; coil; coupling control; power transmission

\section{Introduction}

With the concept of green travel further research, use of electric vehicles has been paid great attention by people, the electric car is using $\mathrm{AC}$ charging as a new energy vehicles, electric vehicles (EV) refers to the vehicle battery as power source, driven by a motor driving wheel, with road traffic safety regulations and requirements the vehicle electric vehicle. Fuel cell is power supply of the automobile. The chemical reaction process of fuel cell will not produce harmful products, but also can realize charging power supply, so the advantages of electric vehicles with environmental protection and energy saving, the zero emission characteristics of electric vehicles, the government of the electric car industry investment and a high degree of attention, the development of electric the car will become a main direction of the motive force of the development of future car. However the charging problem of electric vehicles is not mature, due to charge Station and charging pile Distribution Co., led to the endurance of electric vehicles and convenience is limited, in order to solve the problem of electric vehicle charging a key bottleneck problem, it is necessary to take the charging pile system improvement and the popularity of electric vehicles, charging efficiency optimization of charging pile, reduce charging time and improve the charging power output, improve the distribution range of charging pile, in order to effectively promote the development of electric vehicle industry ${ }^{[1]}$. Therefore, the pile design plays an important role in the development of 
electric vehicle industry of electric vehicle charging.

Electric vehicle charging pile design is the key problem of power transmission optimization control problem, in recent years, pile inductive power transmission control technology in the rapid development of electric vehicle charging, charging pile of scholars on the power transfer efficiency is studied, the power coupling coefficient and transmission efficiency of factors such as transmission and optimization of power control and charging pile closely related to the traditional $\operatorname{method}^{[2]}$, it can optimize the transmission control methods include fuzzy PID control method for electric vehicle charging power, particle swarm optimization of power transmission control method, and adaptive weighted power allocation optimization control method ${ }^{[3,4]}$, the coupling charging pile induction power control of electromagnetic transformer is designed of coil charging pile, charging pile improve power output efficiency, thereby reducing the charging time, and it has obtained better charging pile design results. Among them, the reference [5] analyzed the transmission efficiency optimization of a primary coil and a secondary coil without the alignment of the electric vehicle charging pile, intelligent charging pile optimization design of embedded control system, using an ultra low power ARM processor Cortex-M0 as the kernel, electromagnetic coupling control, improve the output power gain however, this method is not high under the output gain of the interference of electromagnetic radiation of the larger; the reference [6] analyzed the solar and wind power and influence on the charging system into the car, analyzed the electric vehicle induction power response of transmission, wireless charging of the charging pile technology is studied. But the system prospect in engineering application is not clear. In reference [7], the South Korean scholars proposed method of non contact type automobile power transmission system using EECS mechanism The system can realize the power transmission efficiency of $60 \mathrm{KW}$, in the gap of $20 \mathrm{~cm}$, remained at around $80 \%$, but due to the large number of metal brush installed on the vehicle chassis, with the locomotive running distance increases, it will cause the metal brush off the reliability, and the charging system is reduced, resulting in no large-scale commercial applications EECS value.

Aiming at the above problems, a power transmission optimization control technique of electric car charging pile is proposed based on coil magnetic resonance coupling in this paper, the spiral planar coil coupling construction of electric vehicle charging pile is obtained, the design of half bridge LLC resonant inverter as the charging inverter control unit, and then analyzes the resonant frequency, mutual inductance, coil resistance the influence of parameters such as charging pile system power transmission efficiency, and as a basis for parameter optimization design of charging pile coil series, control coil magnetic resonance coupling near resonance, the electricity charging pile optimization design of transmission system. Finally, simulation test is taken, test results show that using the method of pile the design of electric vehicle charging, it has high efficiency of power transmission, to achieve stable output voltage in a large load range, and has high output power gain, it has good application value.

\section{Working principle and design of equivalent circuit of charging pile}

\subsection{Design principle of electric vehicle charging pile}

Electric vehicle charging is taken based on the design of inverter using magnetic resonance mode of charging pile, in the magnetic resonance mode will first input power into high frequency power supply, the energy distribution after installing primary transmission coil series in the charging pile. Then the primary resonance electromagnetic transform by radio links will be delivered to the energy by radio waves the secondary resonance link located in the car battery. The receiving coil to the secondary resonance link energy after magnetoelectric mapping is taken, then the received power after conditioning to the car battery charging. The spiral planar coil as the electric vehicle charging 
magnetic induction coil induction power output, the transmitter coil is fixed on the emission level of the charging pile. The formation of the coil series, the energy transmission through the electromagnetic resonance with the car battery, using spiral planar coil non-contact type structure design method High frequency inverter charging, AC input to the charging pile, in the field as a medium for power transmission, according to the above analysis, the electric vehicle charging structure using the model is shown in Figure 1, schematic diagram of spiral planar coil is shown in Figure 2.

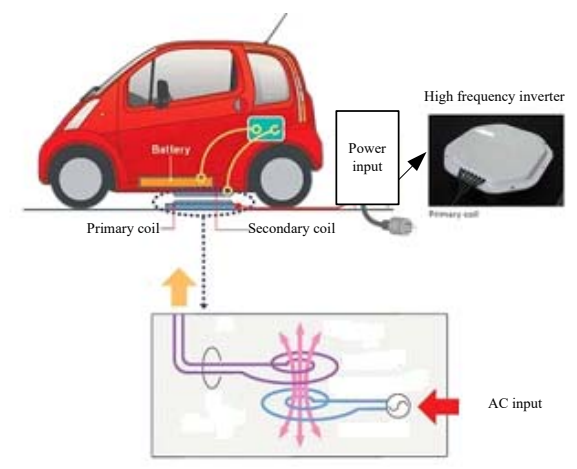

Figure 1 Contactless charging system schematic diagram of spiral planar coil

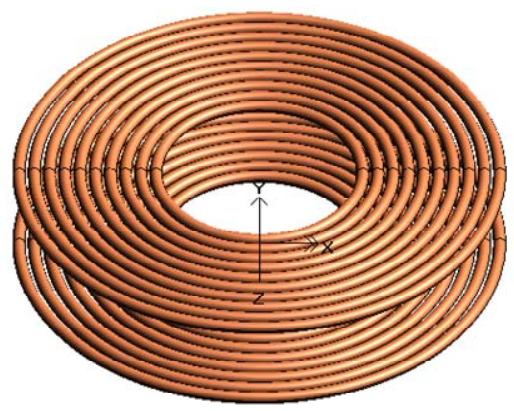

Figure 2 Schematic diagram of spiral planar coil

According to the design principle, the design specifications of electric vehicle charging are designed in this paper: wireless charging by charging pile is designed in this paper, the efficiency of ICPT can do more than $80 \%$, the transmission power is up to $200 \mathrm{~kW}$, it can realize the non-contact electric power transmission in long distance and large scale range. It can be carried out under different conditions of power transmission, and the system efficiency is not affected by the deviation of the coil. In the design process, the charging pile location of the coil is not strict, so it can realize the large vehicles charge. In addition, because the resonance frequency is more stringent, by controlling the frequency of power supply to electric vehicles on the power of different sizes the charging pile is designed in this paper.

In the power transmission design, using magnetic resonance energy transfer mode, the principle diagram of charging piles for power transmission system of electric vehicle charging described is shown in Figure 3, the magnetic resonance of power transmission design, its advantages are as follows:

1) Inverter frequency electromagnetic induction power transmission is generally less than $100 \mathrm{KHz}$, which is determined by the switching frequency of inverter power supply and performance of the magnetic core. The magnetic resonance mode by signal generator and power amplifier power supply, so the emission frequency inverse side energy converter can be greatly improved to $\mathrm{MHz}$; 2) The relative electromagnetic induction type only two coil electromagnetic induction, magnetic resonance energy transfer mode adds 2 resonance link. Resonance coil using distributed capacitance 
to achieve their own resonance to open way. The topological structure of this case system is stable.

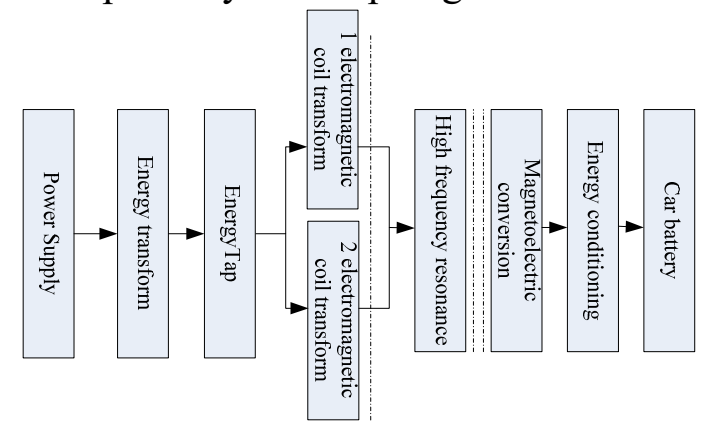

Figure 3 Principle diagram of power transmission system of charging pile

\subsection{Equivalent circuit design}

According to the design principle of the system of charging pile construction, the equivalent circuit diagram of electric vehicle charging pile, this paper adopts $T$ type circuit topology design, by the main circuit, control circuit, detection circuit, auxiliary power supply of four main parts. The main circuit realization of impulse current detection, current transmission filter and rectifier control and inverter control according to the need of load function, inverter circuit design, using AC/DC and DC/DC converter to reverse series switching power supply output. The output current control circuit includes a rectifier filter, inverter and control circuit identification module, according to the switching device in the circuit control mode setting frequency, pulse width and other parameters of model, construction of DC/AC inverter power supply, or through the DC/DC voltage output rectifier voltage detection circuit is realized. The ability of $\mathrm{AD}$ circuit and overload detection and protection is designed, construction of the switching power supply adapter Device, power oscillation detection using 220VAC/220VAC isolation transformer. The auxiliary power supply includes protection circuit, remote software PWM module and power starting module, power supply using UC38 XX series of 8 pin PWM components auxiliary power startup control Vstart/Vcontrol end power remote start and protection, equivalent circuit topology is shown in Figure 4.

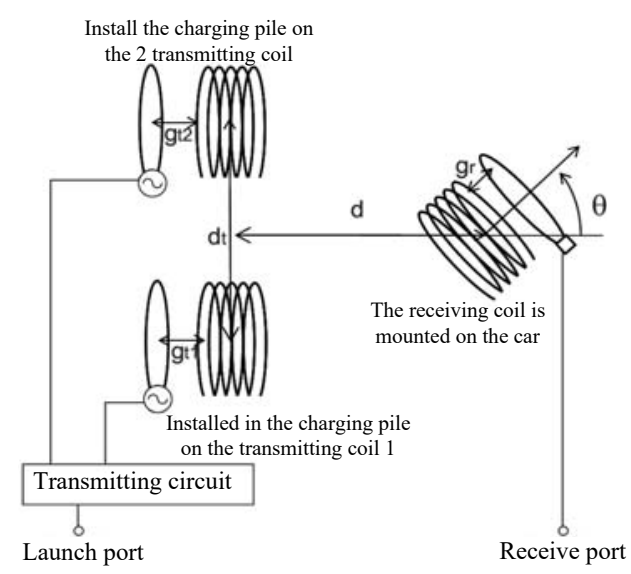

Figure 4 Equivalent circuit topology

According to the equivalent circuit model of Figure 4, the construction of electric vehicle charging pile coupling coil by spiral planar coil is taken, a half bridge LLC resonant inverter is designed as the charging inverter control unit, the coil magnetic resonance coupling control of charging pile is designed.

\section{Coil sequence parameter optimization and power transmission control}

Based on the analysis of the working principle of charging pile and equivalent circuit design in the charging pile, power transmission optimization design, this paper proposes an electric car charging 
coil magnetic resonance coupling power transmission optimization control technique based on mutual inductance frequency analysis, coil resistance parameters to affect the charging pile system power transmission efficiency, includes the establishment of the primary side and secondary side of the transformer model of self inductance and mutual inductance,

Assuming that $L_{11}, L_{22}$ are primary side charging pile spiral planar coil and the secondary winding of the transformer winding inductance, $M$ is mutual inductance between the winding, $\Psi_{1}$ and $\Psi_{2}$ are primary side and secondary side flux, the coupling coefficient is defined as:

$$
k=\frac{M}{\sqrt{L_{11} L_{22}}}
$$

Take the transformer magnetic resonance mode can be calculated by coil sequence, which $\mu_{0}=4 \pi \times 10^{-7}, \quad R_{12}$ is the relative position of the coil, then:

$$
M \cong \frac{\mu_{0}}{4 \pi} \int_{c_{1} c_{2}} \int_{\frac{d s_{1}}{} d s_{2}}^{\left|R_{12}\right|}
$$

Different from the ordinary transformer with high coupling coefficient, self induction power transmission system is much smaller than the leakage inductance. Therefore the self voltage is less than the leakage voltage. It can compensate the voltage leakage caused by the drop in the near the resonance point, and to the model shown in Figure 4 to maintain consistency. It must meet the following conditions:

$$
L_{x}=\left(1-k_{2}\right) L_{11}, L_{m x}=k^{2} L_{11}, \quad n_{x}=k \sqrt{\frac{L_{11}}{L_{22}}}
$$

In order to establish includes DC output performance model, established near resonance current waveform for accurate model including model approximate sine rectifier. The resonant angular frequency and characteristic impedance:

$$
\omega_{0}=\frac{1}{\sqrt{L_{l x} C_{x}}}=2 \pi f_{0}, \quad Z_{0}=\sqrt{\frac{L_{l x}}{C_{x}}}=\omega_{0} L_{l x}=\frac{1}{\omega_{0} C_{x}}
$$

The matching method is used to improve the output power gain by parallel resonant impedance, the initial power input access $220 \mathrm{~V}$, filter capacitor with power supply voltage about 380VDC, UC3843, PWM module, input voltage of electric vehicle charging pile for primary side:

$$
\left[\begin{array}{l}
v_{1} \\
v_{2}
\end{array}\right]=\frac{d}{d t}\left[\begin{array}{l}
\Psi_{1} \\
\Psi_{2}
\end{array}\right]=\left[\begin{array}{cc}
L_{11} & M \\
M & L_{22}
\end{array}\right] \frac{d}{d t}\left[\begin{array}{l}
i_{1} \\
i_{2}
\end{array}\right]
$$

Which, $L_{11}, L_{22}$ are the transmitting frequency and the input of the primary side of the output inductance considering charging pile load for overvoltage protection (OVP), over temperature protection (OTP), except the rectified current is sinusoidal, also considering the situation as follows:

1) The secondary side current at $\theta / \omega_{0}$ time, it is 0 :

$$
i_{\text {sec }}\left(\theta / \omega_{0}\right)=0
$$

2) The rectifier output current equal to the average load current:

$$
\left\langle\left|i_{\text {sec }}(t)\right|\right\rangle=\left\langle i_{0}\right\rangle
$$


3 ) The rectified voltage vi and secondary side coil output voltage difference is equal to the resonant circuit and leakage inductance of the primary voltage and voltage:

$$
v_{i}(t)-n_{x} v_{\text {sec }}(t)=v_{c x}(t)+v_{l x}(t)
$$

The simultaneous solution of the above equations is taken, obtained the resonant current output current and output voltage:

$$
I_{p r i}=\frac{\pi}{4 \omega L_{m x}} \frac{\cos (\alpha)}{\cos (x) \sin (x)} V_{D C}, I_{o}=\frac{2}{\pi} n_{x} \cos (x) I_{p r i}, V_{o}=R_{\text {load }} I_{o}
$$

Wherein:

$$
x=\alpha-\theta=\tan ^{-1}\left(\frac{n_{x}^{2} R_{\text {load }}}{\omega L_{\text {mx }}}\right), \quad \alpha=\tan ^{-1}\left(\frac{\pi^{2}}{8 n_{x}^{2} R_{\text {load }}} \frac{\omega L_{\text {lmx }}-\frac{1}{\omega C_{x}}}{\cos ^{2}(x)}+\tan (x)\right) \quad, \quad \theta=\alpha-x
$$

According to the above the resonant frequency, mutual inductance, coil resistance parameters for the constraint relationship between charging pile system efficiency of power transmission, the optimal design of parameters of charging pile coil series, when the system works in the near resonance, the inverter voltage output for charging pile:

$$
v_{i}(t)=\frac{V_{D C}}{2} \operatorname{sign}[\sin (\omega t)]
$$

According to the above definition, control coil magnetic resonance coupling near resonance, when the primary side current sinusoidal, secondary side output voltage and secondary current are alternately square wave signal, can be expressed as:

$$
i_{p r i}(t) \cong I_{p r i} \sin (\omega t-\alpha)
$$

Considering the secondary side voltage, current flow by $L_{m x}$ is:

$$
i_{L_{m x}}(t)=\frac{1}{L_{m x}} \int_{-\infty}^{t} n_{x} v_{\text {sec }}(t) d t
$$

Synchronous filter capacitor voltage and rectifier diode rectifier input voltage and current, therefore $v_{\mathrm{sec}}$ is:

The secondary side current is:

$$
v_{\text {sec }}(t)=V_{\text {out }} \operatorname{sign}[\sin (\omega t-\theta)]
$$

$$
i_{\text {sec }}(t)=n_{x}\left[i_{p r i}(t)-i_{L_{m x}}(t)\right]
$$

According to the above analysis, the establishment of coil magnetic resonance coupling control model of electric vehicle charging is obtained, power transmission efficiency optimization control output parameter contains the primary side and secondary side control optimization design of self inductance and mutual inductance parameters, control through the coil magnetic resonance coupling, to achieve power charging pile optimization design of transmission system.

\section{Experimental test and analysis}

In order to verify the design of the charging pile in charge of power optimization application performance of transmission control, simulation analysis is taken. Two transmitting coil distance of front and rear wheel wheelbase sedan, about $2500 \mathrm{~m}$. The transmitting coil and the receiving coil diameter of the simulated car tire diameter, the variation range of about $700 \mathrm{~mm}$. and receiving coil transmitting coil distance of $500 \mathrm{~mm} \sim 1000 \mathrm{~mm}$, in order to simulate the vehicle charging performance in the running process, charging pile magnetic resonance power parameters settings for transmission system: 
$L_{p 1}=2.1 \mu \mathrm{H}, L_{p 2}=2.3 \mu \mathrm{H}$, Resonance coil $L_{s}=40.5 \mu \mathrm{H}, L_{r}=55.6 \mu \mathrm{H}, L_{l}=2.1 \mu \mathrm{H}, C_{p 1}=405 p F$, $C_{p 2}=403 p F, C_{s}=12.6 p F, C_{r}=14.6 p F, C_{o}=356 p F, R_{p 1}=45 \Omega, R_{p 2}=44 \Omega, R_{s}=2.5 \Omega, \quad R_{r}=3.2 \Omega$, $R_{o}=30 \Omega, f=27.06 \mathrm{MHz}$. The value of parameter setting of transformer coil resonance is shown in Table 1.

Table 1 Parameter setting of transformer coil resonance

\begin{tabular}{cccccc}
\hline Distance/cm & 40 & 50 & 60 & 70 & 80 \\
\hline Mutual inductance $/ \mu H$ & 1.56 & 1.64 & 1.33 & 0.65 & 0.98 \\
\hline
\end{tabular}

According to the simulation environment and parameter setting, circuit model is built in the laboratory, charging pile test is taken, when the spiral planar coil distance is $80 \mathrm{~mm}$, when the input voltage is $24 \mathrm{~V}$, the output voltage of the inverter by charging pile, the primary side current, the output voltage waveform is shown in Figure 5.

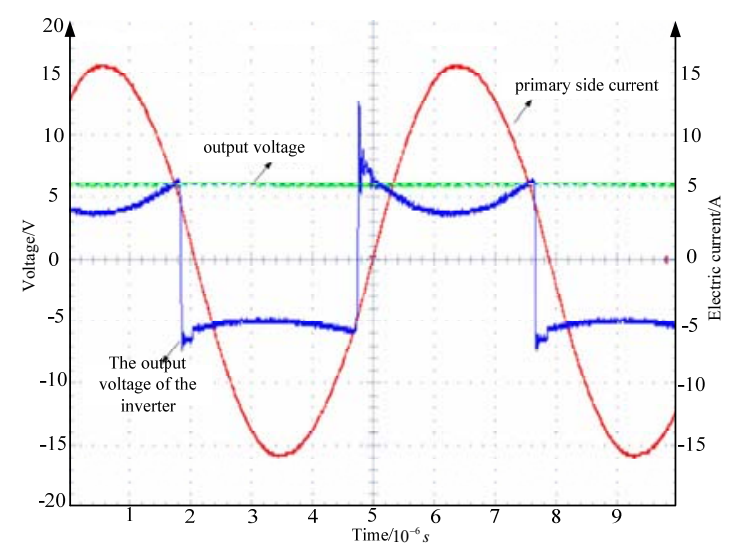

Figure 5 Voltage and current of the charging pile parameter output waveforms

Figure 5 analysis results show that, power transmission control is used by charging pile designed in this paper to transmit, the output voltage is stable, and it has low current oscillation amplitude, it can improve the efficiency of power transmission, Figure 6 shows the voltage gain, charging pile load under the condition of different charging current gain, efficiency and power factor of the test result.

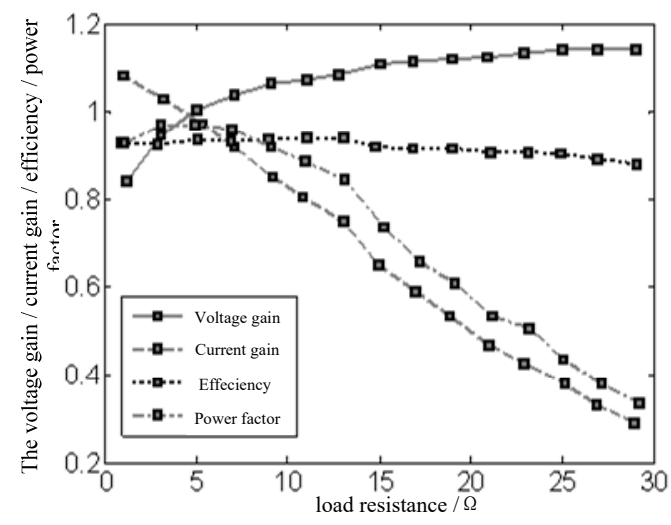

Figure 6 Performance analysis of charging pile

Figure 6 analysis results show that this method for pile design and control of electric vehicle charging, it has high efficiency of power transmission, to achieve stable output voltage in a large load range, output power gain is high, and it can improve the efficiency of charging pile. 


\section{Conclusions}

This paper studies the optimal design problem of charging pile, a power transmission optimization control technique of electric car charging pile is proposed based on coil magnetic resonance coupling., the design of half bridge LLC resonant inverter as charging pile inverter control unit, analyzes the resonant frequency transformer coil resistance coefficient, and other parameters for charging pile system power transmission efficiency, and as a basis for parameter optimization design of charging pile coil series, control coil magnetic resonance coupling near resonance, the electricity charging Pile Optimization Design of transmission system. Test results show that using the method of charging pile design electric vehicle, it has high efficiency of power transmission, it can achieve stable output voltage in a large load range with high charging efficiency and stable charging Voltage.

\section{Acknowledgments}

This project is supported by Foundation for Distinguished Young Talents (Natural Science, 2015KQNCX218)of 2015 Key Platforms and Research Projects of Department of Education of Guangdong Province and 2016 Undergraduate Scientific and Technological Innovation Project Fund of Guangdong Province (pdjh2016b0933).

\section{References:}

[1]LI Ning, LI Yaguang, WANG Hongxing etc. Fuzzy Tracking Control for Fractional-order Permanent Magnet Synchronous Motor Chaotic System[J]. Information and control, 2016,45(1): 8-13.

[2]LU Xinghua, ZHENG Yongtao.Power System Load Forecasting Model Based on Nonlinear Time Series Analysis [J]. Power \& Energy, 2016,37(2): 197-201.

[3]HU CH H, CHEN CH M, SHIAOY SH, et al. Development of a universal contactless charger for handheld devices. IEEE International Symposium on Industrial Electronics[C]// Cambridge, England, 2008: 99-104.

[4]ZHEN Jian-jun,ZHANG Yi,HU Guang-bo. Research on Inductive Power Transmission Technology Based on Spiral Planar Coil[J]. Electrical Automation,2014,36(2): 78-80.

[5]FNATO H, CHIKU Y, HARAKAWAK. Wireless power distribution with capacitive coupling excited by switched mode active negative capacitor[C]/Electrical Machines and Systems (ICEMS), 2010 International Conference, 2010: 117-122.

[6]MAHBOUBI H. Distributed deployment algorithms for efficient coverage in a network of mobile sensors with nonidentical sensing Capabilities[J]. IEEE Transactions on Vehicular Technology, 2014, 63(8): 3998-4016.

[7]SEUNGUOUNG, JOUNGHOA, KIM, Magnetic field design for high efficient and low EMF wireless power transfer in on-line electric vehicle[C]//Antennas and Propagation(EUCAP), Proceedings of the 5th European Conference, 2011: 3979-3982. 